\title{
DETERMINATION OF THE NON-AXISYMMETRIC STRESS STATE IN THE PLASTICITY ZONE BY THE HOLE-DRILLING METHOD WITH OUT-OF-PLANE ESPI
}

\begin{abstract}
A.L. Popov ${ }^{*}$, D.A. Chelyubeev ${ }^{* *}$, A.L. Levitin ${ }^{* * *}$, V.M. Kozintsev ${ }^{* * * *}$, A.V. Chentsov ${ }^{* * * *}$
Abstract: The stress state estimates accounting for plasticity are obtained at residual stress evaluation performed by hole-drilling method and out-of-plane ESPI. The plasticity zone in the sample is present prior to the probe hole drilling. The sample is a steel composite disc, assembled by fitting rings on the plug. The initial stress distribution in the composite elastic-ideal plastic disk accounting for the formation of a plastic zone in the covering ring is obtained by the solution of Gadolin in elastoplastic formulation. A simple analytical algorithm to adjust the non-axisymmetric stresses in comparison with the calculation according to the elastic model is developed. The case of probe hole drilling in the area of plastic deformations is considered.
\end{abstract}

Keywords: Nonaxisymmetric Stress, Hole-drilling Technique, Out-of-plane Speckle Interferometry, Plasticity Effects, FEM.

\section{Introduction}

The hole method proposed in 1932 (Mathar, 1934) is presently generally accepted in the diagnosis of residual stress-strain state as a low-damaging method for measuring stress-strain state (Chernyshev, 1996 and Schajer, 2010). Two main methods of detecting deformations and microdisplacements released by a hole in a stressed body have formed: the tensometric and optical with the prevalence of laser-holographic and speckle-interferometric measurement technologies. In the latter case, the optical method is subdivided according to the measured components of displacements in the vicinity of the hole: in the plane of the observed body surface (Nelson, 1986 and Zhang, 1998), or along the normal to it (Chernyshev, 1996, Lin, 1994 and Goldstein, 2010). In comparison with strain gauging, laser-interferometric methods have the advantage of non-contact and measurement efficiency. Measuring the normal displacement component in stress evaluation has additional advantages over measuring tangential components: 1 ) more simple optical scheme of the interferometer, 2) determining the directions of the main stresses directly from the interference pattern, rather than measuring from tangential components when the displacement is determined along only one of the axes not always coinciding with the direction of the main stress (Lin, 1994) 3) simple provision of the maximum theoretical sensitivity using the illumination of an object along the normal to its surface, while achieving the maximum theoretical sensitivity in the interferometric

\footnotetext{
Prof. Alexander L. Popov, DSc.: Ishlinsky Institute for Problems in Mechanics of the Russian Academy of Sciences, Prospekt Vernadskogo, 101-1, Moscow 119526; Russia, National Research University, Moscow State University of Civil Engineering, Moscow; Russia, popov@ipmnet.ru

** Researcher Dmitri A. Chelyubeev: Ishlinsky Institute for Problems in Mechanics of the Russian Academy of Sciences, Prospekt Vernadskogo, 101-1, Moscow 119526; Russia

*** Leading Programmer Alexander L. Levitin: Ishlinsky Institute for Problems in Mechanics of the Russian Academy of Sciences, Prospekt Vernadskogo, 101-1, Moscow 119526; Russia, alex_lev@ipmnet.ru

***** Senior Researcher Viktor M. Kozintsev, PhD.: Ishlinsky Institute for Problems in Mechanics of the Russian Academy of Sciences, Prospekt Vernadskogo, 101-1, Moscow 119526; Russia

****** Senior Researcher Alexander V. Chentsov, PhD.: Ishlinsky Institute for Problems in Mechanics of the Russian Academy of Sciences, Prospekt Vernadskogo, 101-1, Moscow 119526; Russia, chentsov@ipmnet.ru
} 
method for measuring planar deformations, seems to be almost impossible (Zhang, 1998): sensitivity is proportional to the sine of object illumination rays incidence angle, and illumination of the sample is greatly reduced if this angle exceeds $60^{\circ}$ from the normal.

Along with the advantages over non-destructive methods, the hole method has several shortcomings, the main one being the stress concentration near the probe hole, which causes plasticity in its vicinity at high values of residual stress. As a result, the existing standards (GOST R 52891-2007 and ASTM E837-13a, 2013) for the measurement of stresses by the hole method, which use the linear theory of elasticity to determine the residual stresses, set limits on the measured stresses at $0.4-0.7$ of the materials yield strength.

To overcome this limitation, a series of numerical studies on three-dimensional problems for blind holes (Lin, 1995, Moharami, 2008, Beghini, 2010 and Seifi, 2012), taking into account the plasticity effect that are useful for the various types of strain gauges used in the standard (ASTM E837-13a, 2013), has been recently performed. These studies, as some other works, report that neglecting the effect of plasticity can lead to the error in stress determination up to 35\% (13-18\%, on average).

The obtained results, however, were related only to the relations of stresses and tangential deformations recorded by strain gauges and laser interferometric optical methods adjusted for this type of measurement. They didn't use the data on normal body surface movements released after drilling the hole, the distributions of which are just necessary for the speckle-interferometric method for determining residual stresses according to the standard (GOST R 52891-2007).

The state of the topic required improvements and solution of the corresponding three-dimensional elastoplastic problems, as well as specific experimental studies which results would ensure the improvement of the speckle-interferometric method for recording residual stresses along the normal components of displacements in the vicinity of the probe hole.

Such studies were performed in (Popov, 2018) for the case of creating a hole in the body with residual axisymmetric stresses within the elastic range. The present study considers the effect of plasticity, when a probe hole is created in the zone of plasticity that is formed in the sample before the creation of this hole.

\section{Samples and methods of study}

The samples were steel assembled disks. They were created by fitting the covering rings on plugs. Each sample was manufactured with significant axisymmetric stresses in the plug and large nonaxisymmetric stresses of opposite sign in the outer ring. Such combinations of stresses are often encountered in the analysis of residual stresses after welding. Note that although the experimental samples of this type presented in (Hsu, 1982) have been previously used in such testing, they were designed and made of aluminum alloy, so that there were stresses much lower than the plastic limit, and with such reserve that after drilling the probe hole, the stress concentration did not increase the maximum stress level in the samples beyond the plastic limit (Steinzig, 2001 and Ribeiro, 2009).

In this work, steel composite disks with stresses on the plugs close to the yield point were made. Ring shaped plastic zones near the contact line with the plugs were formed in the covering rings during the manufacturing process.

The design of such samples required solution of Gadolin problems in the elastoplastic formulation, correcting the data of the original elastic stress distribution before landing with the specified preload of cylindrical bodies. The known publication on characteristics of fitting accounting for elastoplastic properties of materials is devoted mostly to studies of the effects of stress state variation in the joined bodies at various dependencies of yield stress on temperature. At the same time, they were carried out at plain strain under the Tresca-Saint-Venant yield condition. In this work and in (Bukhalov, 2018) the study is based on the model of plane stress under the condition of von Mises yield. The yield strengths of materials of the connected bodies in the selected fitting process with cooling of the plug in liquid nitrogen $\left(-196^{\circ} \mathrm{C}\right)$ and heating the covering ring to $150^{\circ} \mathrm{C}$ were almost equal to the values at $20^{\circ} \mathrm{C}$. This, as well as the nonlinearity factor of the elastoplastic state, is in favor to the fact that the results of this work in terms of the solution of the elastic-plastic Gadolin problem cannot be derived from the results obtained by other authors. For fig. 1 the results of this statement are presented in the form of dependencies of the radial and circumferential dependencies of the radial and circumferential stress components in the covering ring on 
the distance to the plug on the section of the ring adjacent to the plug, calculated for the elastic $\left(\sigma_{r}^{\mathrm{e}}, \sigma_{\theta}^{\mathrm{e}}\right)$ and elastic-plastic $\left(\sigma_{r}^{\mathrm{p}}, \sigma_{\theta}^{\mathrm{p}}\right)$ case. Ring parameters are: yield strength $\sigma_{\mathrm{Y}}=300 \mathrm{MPa}$, outer diameter $d_{\mathrm{r}}=130 \mathrm{~mm}$, diameter of the joint contour and the plug $d_{\mathrm{p}}=38 \mathrm{~mm}$. The yield strength of the plug material $\sigma_{\mathrm{Y}}=340 \mathrm{MPa}$, which is higher than that of the ring material. The interference fit when fitting was consistent with the contact pressure $0.9 \sigma_{\mathrm{Y}}$. The $\sigma_{r}^{\mathrm{p}}, \sigma_{\theta}^{\mathrm{p}}$ are FEM curves and show the corresponding functions from the numerical solution of three-dimensional elastic-plastic problem.

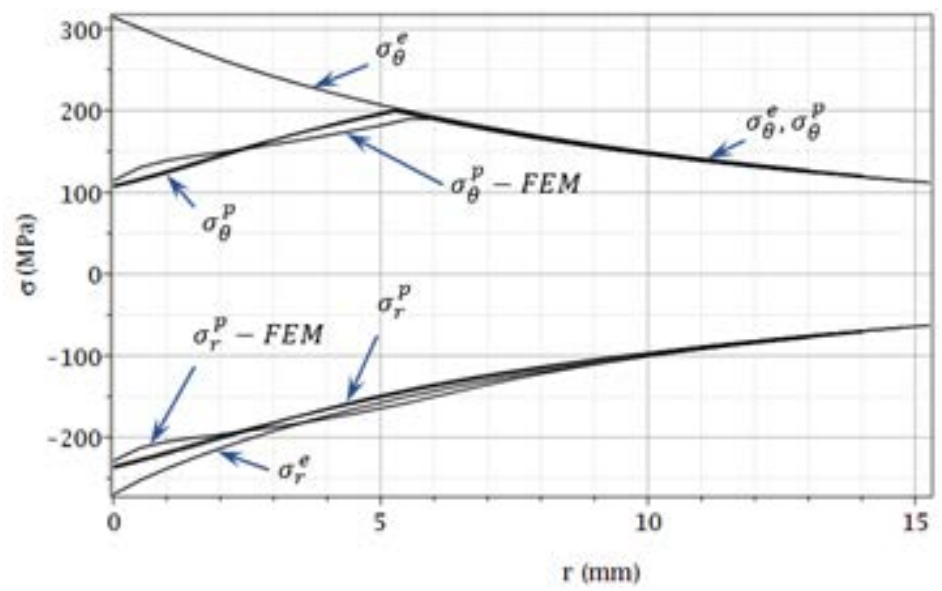

Fig. 1: Distribution of circumferential and radial stresses in an elastic-plastic covering ring.

Figure 1 shows that the compressive radial stresses calculated on the elastic and elastic-plastic models do not differ significantly all the way from the plug to the outer edge of the composite disk. At the same time, the ring-liner interface has more than two-fold difference in the circumferential stresses calculated on these models.

When removed from the plug, this difference gradually decreases and ends at a distance coinciding with the radius of the plastic zone boundary calculated by the criterion of equality of the equivalent stress to yield strength of the ring material:

$$
\sigma_{\text {eq }}=\sqrt{\sigma_{r}^{2}-\sigma_{r} \sigma_{\theta}+\sigma_{\theta}^{2}}=\sigma_{\mathrm{Y}}
$$

Therefore, drilling the probe hole in the initially plastic zone and determining the residual stresses by the conventional elastic model (Chernyshev, 1996) can create a large error in the circumferential stress.

To correct the error caused by the calculation of the circumferential stress by the elastic model when the probe hole enters the plastic zone, the following approach is proposed. The circumferential and radial stresses found from the elastic calculation are substituted into the formula for the equivalent stress. If it turns out that $\sigma_{\text {eq }}>\sigma_{\mathrm{Y}}$ the value $\sigma_{r}$ is taken to be equal to the value calculated in the assumption of elasticity of the material, since it is known that the equivalent stress cannot exceed the yield point. Then $\sigma_{\theta}$ is determined from the yield condition in the absence of hardening:

$$
\sigma_{\theta}=\frac{\sigma_{r}}{2}+\frac{1}{2} \sqrt{4 \sigma_{\mathrm{Y}}^{2}-3 \sigma_{r}^{2}}
$$

The specific calculation performed at the location of the center of the probing hole with a diameter of $3 \mathrm{~mm}$ and a depth of $3 \mathrm{~mm}$ at a distance of $2 \mathrm{~mm}$ from the plug. The calculation of stresses using an elastic model gave the following results: $\sigma_{\mathrm{r}}=-200 \mathrm{MPa}, \sigma_{\theta}=260 \mathrm{MPa}$.

The equivalent stress determined from (1) was $400 \mathrm{MPa}$, which is greater than the yield strength of the ring material. This means that the probe hole is created in the plasticity zone and the circumferential stress is determined with an error.

Assuming that the calculated radial stress component is determined by the elastic model with sufficient accuracy and substituting its value in (2), we find the corrected value of the circumferential stress: $\sigma_{\theta}=145 \mathrm{MPa}$, which is less than one percent different from the true value of the circumferential stress at this point. 


\section{Conclusions}

The effect of plasticity in determining the residual stresses using the hole method is most evident when the probe hole enters the plastic zone. We proposed a simple analytical algorithm that by the results of applying the elastic model determines the probe hole entering into the area of plastic deformations and corrects the values of the non-axisymmetric stresses in comparison with a calculation according to the elastic model.

\section{Acknowledgement}

This work was partially supported by Russian State Assignment under contract No. AAAA-A17-117021310386-3 and the Russian Foundation for Basic Research (RFBR) under Grant

No. 19-01-00100.

\section{References}

ASTM E837-13a (2013) Standard Test Method for Determining Residual Stresses by the Hole-Drilling Strain-Gage Method, $16 \mathrm{p}$.

Beghini, M., Bertini, L. and Santus, C. (2010) A procedure for evaluating high residual stresses using the blind hole drilling method, including the effect of plasticity. J. Strain Analysis, 45, pp 301-318.

Bukhalov V. I., Popov A. L., Chelyubeev D. A. (2018) Gadolin's problem in an elastoplastic formulation. Prikladnaya Matematika i Mekhanika, 82, 6, pp 808-816 (in Russian).

Chernyshev, G. N., Popov, A. L., Kozintsev, V. M., et al. (1996) Residual stresses in deformable solids. Fizmatlit, Moscow (in Russian).

Goldstein, R. V., Kozintsev, V. M. and Popov, A. L. (2010) Using an electronic speckle interferometry for measurement of a stress-deformation state of elastic bodies and structures In: Recent Advances in Mechanics: Selected Papers from the Symposium on Recent Advances in Mechanics, Acad. of Athens, Athens, Greece, 17-19 Sept., 2009. Springer, pp 191-206.

GOST R 52891 (2007) Control of residual technological stresses by laser interferometrical method general requirements (in Russian).

Hsu, N., Proctor, T. and Blessing, G. (1982) An Analytical Approach to Reference Samples for Ultrasonic Residual Stress Measurement. J. Test. and Eval., 10, 5, pp 230-234.

Lin, S. T., Hsieh, C. T. and Hu, C. P. (1994) Two holographic blind-hole methods for measuring residual stresses. Exp. Mech., 34, 2, pp 141-147.

Lin, Y. C. and Chou, C. P. (1995) Errors induced by local yielding around hole-drilling method for measuring residual stress of material. J. Mater. Sci. Technol., 11, pp 600-605.

Mathar, J. (1934) Determination of initial stresses by measuring the deformation around drilled holes Trans ASME, 56, 4, pp 249-254.

Moharami, R and Sattari-Far, I. (2008) Experimental and numerical study of measuring high welding residual stresses by using the blind-hole-drilling technique J. Strain Analysis, 43, pp 141-148.

Nelson, D. V., McCrickerd J. T. (1986) Residual stress determination through combined use of holographic interferometry and blind-hole drilling. Exp. Mech., 26, 4, pp 371-378.

Popov, A. L., Alexandrov, S. E., Kozintsev, V. M., Levitin, A. L., Chelyubeev, D. A. (2018) Effect of plasticity at out-of-plane electronic speckle pattern interferometry diagnostics of axisymmetric stresses by the hole-drilling method. J. Strain Anal. Eng., 53, 1, pp 3-14.

Ribeiro, J. et al. (2009) Measurement of Residual Stresses with Optical Techniques. Strain, 45, pp 123-130.

Schajer, G. S. (2010) Hole-drilling residual stress measurements at 75: origins, advances, opportunities. Exp. Mech., 50, pp 245-253.

Seifi, R. and Salimi-Majd, D. (2012) Effect of plasticity on residual stresses measurement by hole drilling method. Mech. Mater., 53, 72-79.

Steinzig, M., Hayman, G., Prime, M. B. (2001) Verification of a Technique for Holographic Residual Stress Measurement. In: Residual Stress Measurement and General Nondestructive Evaluation / PVP Vol. 429, Presented at the 2001 ASME Pressure Vessels and Piping Conference, Atlanta, GA July 23-26, 2001, pp 65-70.

Zhang, J. (1998) Two-dimensional in-plane electronic speckle pattern interferometer and its application to residual stress determination. Opt. Eng., 37, pp 2402-2409. 